\title{
HIDRÓlISE ASSISTIDA POR ULTRASSOM DO ÓLEO DE CRAMBE (Crambe abyssinica) EM MEIO LIVRE DE SOLVENTE ORGÂNICO
}

\author{
B. T. F. MELLO ${ }^{1}$, G. M. RODRIGUES ${ }^{1}$ e C. SILVA $^{1}$ \\ ${ }^{1}$ Universidade Estadual de Maringá, Departamento de Tecnologia \\ E-mail para contato: camiladasilva.eq@gmail.com
}

RESUMO - O presente trabalho teve como objetivo a obtenção de hidrolisado, rico em ácidos graxos livres (AGLs), a partir do óleo de Crambe. Os experimentos foram conduzidos em banho de ultrassom, utilizando a enzima Lipozyme ${ }^{\circledR}$ TL IM como catalisador em meio livre de solvente orgânico. Os experimentos avaliaram o efeito do teor de água, temperatura, percentual de catalisador e tempo de reação. Os resultados obtidos reportam maiores rendimentos com adição de $40 \%$ de água (em relação à massa de óleo) e temperatura de $45^{\circ} \mathrm{C}$. O percentual de catalisador favorece a reação até percentuais de $10 \%$ (em relação à massa de substratos), sendo que acima deste percentual não é observada diferença significativa no rendimento $(\mathrm{p}>0,05)$, para as reações conduzidas em 4 horas. O efeito do tempo reacional relatou que as reações conduzidas com tempos acima de 2 horas não ocasionam o aumento no teor de AGLs.

\section{INTRODUÇÃO}

A busca por matéria-prima de baixo custo e sem fins alimentícios para a produção de biodiesel tornou-se necessária do ponto de vista tecnológico e econômico. O Crambe (Crambe abyssinica) surgiu como uma boa opção, por ser uma planta com alto teor de óleo, com uma cultura de ciclo curto, em média 90 dias, e com produtividade entre 1.000 e 1.500 quilogramas por hectare (Silva et al., 2013). As sementes de Crambe possuem 35-60\% de óleo em sua semente e 20-40\% de proteína. Os ácidos graxos predominantes em sua semente são: erúcico (58-66\%) e oleico $(17,2 \%)$ tornando o óleo impróprio para o consumo humano e tendo seu cultivo focado na produção de biodiesel (Maciel et al., 2014).

Recentemente, é proposta a produção de biodiesel por hidroesterificação, para substratos com elevada acidez e umidade, que consiste na hidrólise dos triglicerídeos seguida da esterificação dos ácidos graxos obtidos (Meng et al., 2011; Oliveira et al., 2011). Como cerca de 80\% do custo de produção do biodiesel é proveniente do custo da matéria-prima, a hidroesterificação permite um significativo salto na viabilidade de um projeto para produção de biodiesel (Hass et al., 2006; Ranganathan et al., 2008). Portanto, ao invés de diminuir a acidez de uma matéria-prima através de um refino, a hidrólise aumenta propositadamente a acidez da mesma. Após a hidrólise, os ácidos graxos gerados são então esterificados, obtendo-se os ésteres de ácidos graxos (Meng et al., 2011; Oliveira et al., 2011). 
$\mathrm{Na}$ produção de biodiesel por hidroesterificação, a catálise heterogênea enzimática tem como principais atrativos a facilidade de separação do catalisador do processo e a pureza do glicerol gerado como subproduto, além da possibilidade de reutilização do catalisador no processo (Ranganathan et al., 2008). A hidrólise enzimática tem algumas vantagens sobre a química, uma vez que requerem temperaturas mais baixas, que previne a degradação dos produtos e reduz os custos energéticos (Antczak et al., 2009).

A reação dos triglicerídeos com água (hidrólise) ocorre na interface entre as duas fases, uma vez que estes substratos são imiscíveis entre si. O contato entre as duas fases é usualmente promovida por agitação mecânica, especialmente a nível industrial, mas recentemente têm-se tentado solucionar este problema através da aplicação do ultrassom (Yu et al., 2010; Feiten et al., 2014). Nas reações assistidas por irradiação ultrassônica, as cavitações (formação, aumento e implosão de bolhas no meio reacional) geradas pelo ultrassom, aumentam a miscibilidade entre os reagentes, aumentando a transferência de massa entre as duas fases através do fornecimento de aquecimento e de mistura. A cavitação provoca um aumento localizado de temperatura na fronteira das fases e fornece a energia mecânica para a mistura e a energia de ativação necessária para iniciar a reação. $\mathrm{O}$ colapso das bolhas de cavitação rompe a fronteira de fase e promove a emulsificação por jatos ultrassônicos. Estes efeitos aceleram a velocidade da reação e encurtar a sua duração (Thanh et al., 2010).

Dentro do contexto descrito, este trabalho está focado na obtenção de hidrolisado do óleo de crambe, rico em AGLs, a ser utilizados em reações de esterificação. Os experimentos foram conduzidos em banho de ultrassom avaliando o efeito das variáveis operacionais (percentual de água, temperatura, percentual de catalisador e tempo de reação) no rendimento em AGLs,

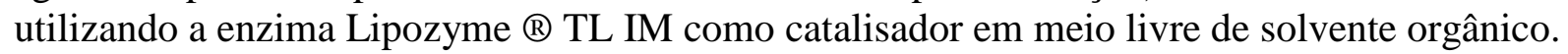

\section{MATERIAIS E MÉTODOS}

\subsection{Materiais}

Nas reações de hidrólise foram utilizados óleo de Crambe (Fundação MS) e como catalisador a enzima Lipozyme® TL IM. A composição química do óleo usado neste trabalho encontra-se reportada na Tabela 1 . O teor de AGLs (mg $100 \mathrm{mg}^{-1}$ ) e conteúdo de água (\% mássico) foi determinado em aproximadamente $1,88 \pm 0,1$ e 0,016 , respectivamente. Na etapa de titulação das amostras foram utilizados: solução de éter etílico:álcool etílico 2:1 (v:v) (Vetec/Nuclear), hidróxido de sódio $0,25 \mathrm{~mol} \mathrm{~L}^{-1}$ (Nuclear) e o indicador fenolftaleína (Nuclear). 
Tabela 1 - Composição química do óleo de crambe.

\begin{tabular}{|c|c|}
\hline Ácido Graxo & Percentual (\%) \\
\hline Palmítico (C16:0) & $1,950 \pm 0,138$ \\
\hline Palmitoleico (C16:1n-7) & $0,159 \pm 0,012$ \\
\hline Esteárico (C18:0) & $0,980 \pm 0,038$ \\
\hline Oleico (C18:1n-9) & $17,434 \pm 0,604$ \\
\hline cis-vacenico (C18:1n-7) & $0,460 \pm 0,041$ \\
\hline Linoleico (C18:2n-6) & $7,466 \pm 0,260$ \\
\hline Linolenico (C18:3n-3) & $4,232 \pm 0,591$ \\
\hline Araquídico (C20:0) & $1,167 \pm 0,002$ \\
\hline Eicosadienoico (C20:2n-6) & $1,081 \pm 0,011$ \\
\hline Erúcico (C22:1n-9) & $58,394 \pm 0,005$ \\
\hline
\end{tabular}

\subsection{Procedimentos}

As reações foram conduzidas em um Erlenmeyer de vidro de $50 \mathrm{~mL}$, conectado a um banho ultrassônico com controle da temperatura (Ultronique, Q 5.9 /40A) e agitador mecânico (IKA RW 20 D). A enzima foi colocada em estufa a $40{ }^{\circ} \mathrm{C} / 1 \mathrm{~h}$ para ativação. Os substratos (óleo vegetal e água) foram adicionados ao reator, sendo o mesmo acoplado ao banho ultrassom dando inicio ao aquecimento. Após atingida a temperatura de reação, a enzima era adiciona ao meio e iniciada a agitação do sistema e o fornecimento de irradiação ultrassônica. Os experimentos foram realizados com agitação fixa em $600 \mathrm{rpm}$ e potência máxima do banho ultrassônico $(132 \mathrm{~W})$, tais condições foram definidas baseadas no trabalho de Feiten et al. (2014). No final da reação, a enzima foi removida por filtração à vácuo, e os produtos foram recuperados para posterior análise. As amostras sem a enzima foram transferidas para frascos de amostragem e colocados em rota evaporador à vácuo para a evaporação do excesso de solvente. Por fim, as amostras foram refrigeradas para posterior análise.

O percentual de ácidos graxos livres foi determinado com base no método Ca 5a-40 (AOCS; 1998), que se baseia no método de titulação ácido-base, utilizando uma solução etanólica de hidróxido de potássio $(\mathrm{KOH})$ previamente padronizada $\left(0,25 \mathrm{~mol} \mathrm{~L}^{-1}\right)$ como titulante. Cada amostra foi titulada em duplicata e o teor de AGLs nas amostras foi calculada a partir da equação 1: 


$$
\text { Teor de AGLs (\%) }=\frac{\text { V.C.M }}{10 . \mathrm{m}}
$$

onde $\mathrm{V}$ corresponde ao volume da solução utilizada para a titulação em $\mathrm{mL} ; \mathrm{M}=$ massa molar do ácido oleico $\left(282 \mathrm{~g} \mathrm{~mol}^{-1}\right), \mathrm{C}=$ concentração do titulante $\left(\mathrm{mol} \mathrm{L}^{-1}\right)$ e $\mathrm{m}$ é a massa da amostra em gramas $(\mathrm{g})$.

Os dados foram submetidos à análise de variância, analisados pela ANOVA e teste de Tukey $(\mathrm{p}>0,05)$ para avaliar as diferenças das médias dos tratamentos.

\section{RESULTADOS E DISCUSSÃO}

\subsection{Efeito do Teor de Água}

O efeito o teor de água (em relação à massa de óleo) sobre a hidrólise de óleo de crambe foi avaliada, mantendo-se a temperatura fixa em $65{ }^{\circ} \mathrm{C}$, percentual de catalisador (em relação aos substratos) a 10\%, e tempos de reação de 2 e 4 horas (Figura 1). A Figura 1 mostra que o aumento da quantidade de água no meio reacional, na faixa de 20 a 40\%, favorece a produção de AGLs. No entanto, quando um excesso de água foi aplicado ao meio (50\%), não houve nenhum efeito significativo sobre o rendimento $(\mathrm{p}>0,05)$ nas condições avaliadas.

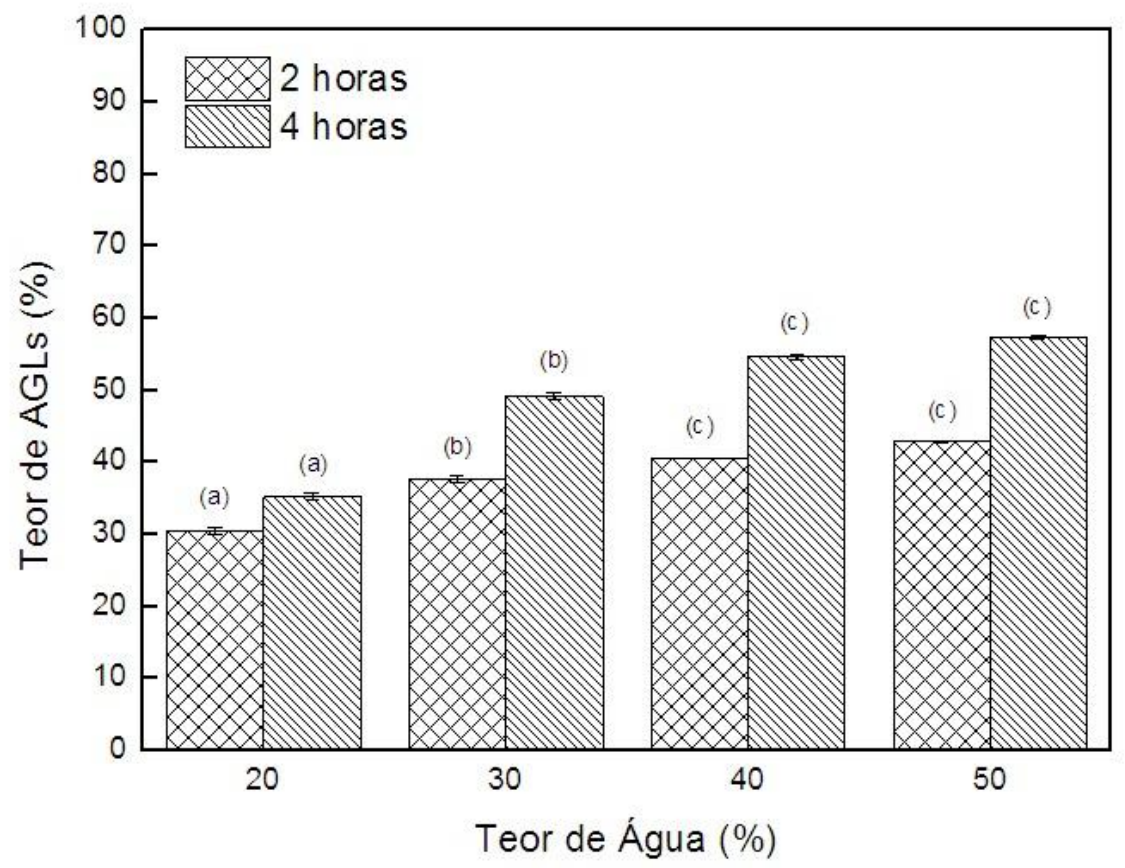

Figura 1 - Efeito do teor de água na hidrólise do óleo de crambe à $65{ }^{\circ} \mathrm{C}$ e percentual de catalisador de $10 \%$. Médias seguidas de mesmas letras (para um mesmo tempo de reação), em cada coluna, não se diferem estatisticamente $(\mathrm{p}>0,05)$. 
Liu et al. (2008), ao investigarem o efeito do ultrassom na hidrólise enzimática de óleo de soja, relatam que maiores quantidades de água no meio reacional resultam no aumento da produção de AGLs. Utilizando 10\% de água, 17\% do óleo foi hidrolisado em AGLs, enquanto que, ao aumentar-se a proporção para $25 \%$, um hidrolisado com $33 \%$ de AGLs foi obtido. Na produção do concentrado natural de beta-caroteno a partir de óleo de buriti por hidrólise enzimática, Ribeiro et al. (2012) observaram o acréscimo nos rendimentos em AGLs de 56,6\% para $62,9 \%$ utilizando razões mássicas de óleo:água 14 e $26 \%$, respectivamente utilizando a enzima TL IM (imobilizada). Raspe et al. (2013) reportam o efeito significativo do percentual de água de 10 a 50\% no meio reacional de hidrólise do óleo de macaúba, utilizando a enzima Lipozyme® TL IM.

\subsection{Efeito da Temperatura}

A Tabela 2 apresenta o efeito da temperatura, avaliada no intervalo de 35 a $65^{\circ} \mathrm{C}$, utilizando $40 \%$ de água, percentual de catalisador (em relação à massa dos substratos) de $10 \%$ e 4 horas de reação. A partir da Tabela 2, pode-se observar que o aumento da temperatura aumenta a produção de AGLs no intervalo de 35 a $45^{\circ} \mathrm{C}$, obtendo teores de 58,6 e 62,76\% de AGLs, respectivamente. Com o aumento da temperatura para 55 e $65^{\circ} \mathrm{C}$ ocorre o decréscimo na produção de AGLs. Feiten et al. (2014) relatam que a temperatura de $65^{\circ} \mathrm{C}$ é a ótima para utilização da enzima Lipozyme ${ }^{\circledR}$ TL IM, no entanto, neste trabalho melhor rendimento foram obtidos na temperatura de $45^{\circ} \mathrm{C}$. Awadallak et al. (2013) reportam que a utilização do ultrassom leva a evaporação da água disponível no meio reacional. Desta forma, acredita-se que o aumento da temperatura eleve as taxas de evaporação de água, ocasionando o decréscimo no teor de AGLs.

Tabela 2 - Efeito da temperatura na hidrólise do óleo de crambe utilizando $40 \%$ de água, percentual de catalisador de $10 \%$ e tempo de 4 horas. Médias seguidas de mesmas letras, em cada coluna, não se diferem estatisticamente ( $p>0,05)$.

\begin{tabular}{|c|c|}
\hline Temperatura $\left({ }^{\circ} \mathrm{C}\right)$ & Teor de AGLs (\%) \\
\hline 35 & $58,63 \pm 0,49^{\mathrm{a}}$ \\
\hline 45 & $62,75 \pm 0,08^{\mathrm{b}}$ \\
\hline 55 & $60,76 \pm 0,25^{\mathrm{c}}$ \\
\hline 65 & $54,70 \pm 0,37^{\mathrm{d}}$ \\
\hline
\end{tabular}

\subsection{Efeito do Percentual de Catalisador}

A Tabela 3 apresenta os resultados dos experimentos realizados, da variação da concentração de catalisador de 5 a $15 \%$ (em relação à massa de óleo) obtidos à $45^{\circ} \mathrm{C}, 40 \%$ de água e 4 horas de reação. Pode-se observar a partir da Tabela 3, que o aumento no percentual de catalisador, de 5 a 10\%, conduz à um aumento da produção de AGLs, de 54,9\% à 62,8\%. No entanto, teores acima de $10 \%$ não apresentam influência significativa na obtenção de AGLs ( $p>0,05)$, nas condições avaliadas. Isto pode ser explicado devido à saturação da 
enzima na interface entre o óleo e a fase aquosa, onde o aumento da concentração da enzima não ocorre alterações significativas na taxa de reação (Rooney e Weatherly, 2001).

Tabela 3 - Efeito do percentual de catalisador no rendimento da hidrólise do óleo de crambe à 45 ${ }^{\circ} \mathrm{C}, 40 \%$ de água e 6 horas de reação. Médias seguidas de mesmas letras, em cada coluna, não se diferem estatisticamente $(\mathrm{p}>0,05)$.

\begin{tabular}{|c|c|}
\hline Catalisador (\%) & Teor de AGLs (\%) \\
\hline 5 & $54,97 \pm 0,14^{\mathrm{a}}$ \\
\hline 7.5 & $61,06 \pm 0,17^{\mathrm{b}}$ \\
\hline 10 & $62,76 \pm 0,09^{\mathrm{c}}$ \\
\hline 12.5 & $60,74 \pm 0,91^{\mathrm{c}}$ \\
\hline 15 & $62,22 \pm 0,75^{\mathrm{c}}$ \\
\hline
\end{tabular}

\subsection{Efeito do Tempo de reação}

O efeito do tempo sobre a hidrólise de óleo de crambe foi avaliada mantendo-se a temperatura fixa em $45{ }^{\circ} \mathrm{C}$, percentual de catalisador (em relação aos substratos) de 7,5 e $10 \%$, $40 \%$ de água e tempos de reação de 1, 2, 4, 6 e 8 horas (Figura 2).

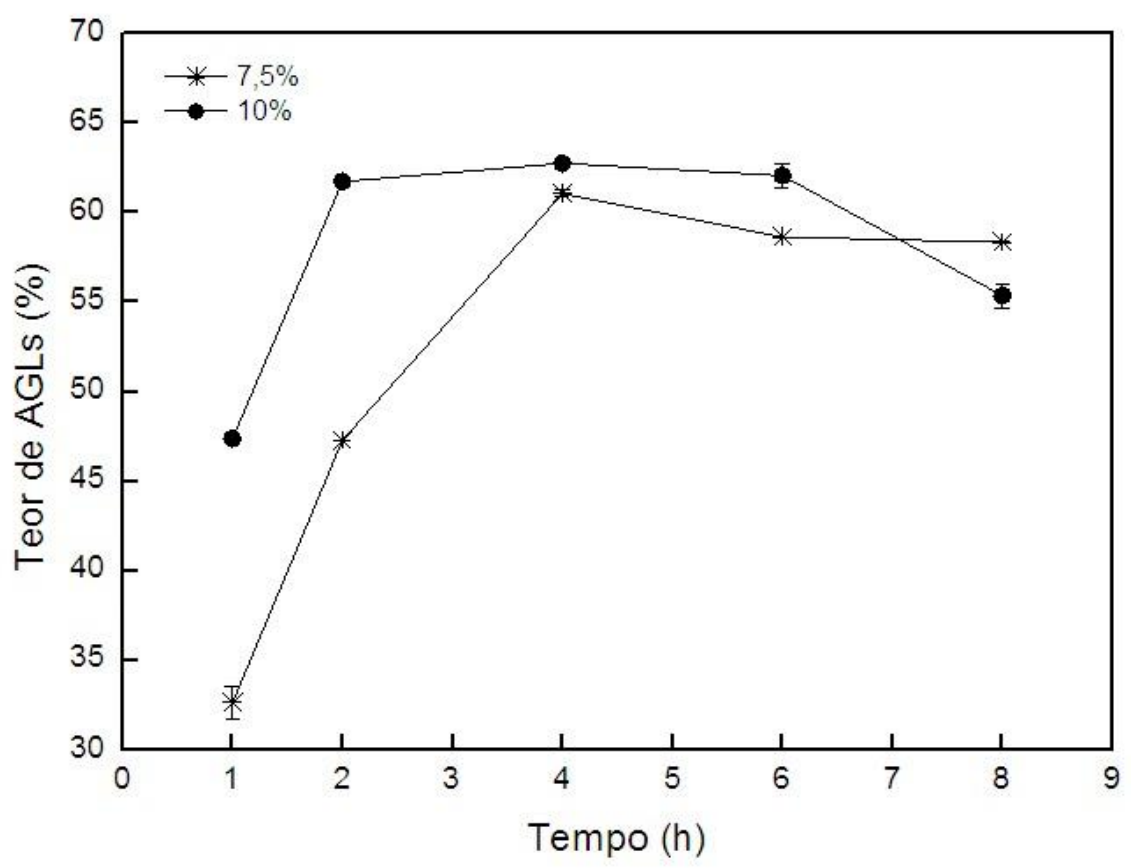

Figura 2 - Efeito do teor de água na hidrólise do óleo de crambe, à $65{ }^{\circ} \mathrm{C}$ e percentual de catalisador de $10 \%$. Médias seguidas de mesmas letras (para um mesmo tempo de reação), em cada coluna, não se diferem estatisticamente ( $p>0,05)$. 
Os resultados apresentados na Figura 2 demonstram que tempos maiores que 2 horas não ocasionam o aumento do teor de AGLs e no tempo de 8 horas um pequeno decréscimo no rendimento em AGLs é observado, o que também foi relatado no trabalho de Feiten et al. (2014). Observa-se ainda, nos dados da Figura 2, o efeito mais pronunciado da concentração de catalisador nas reações conduzidas em 1 e 2 horas, evidenciando o efeito desta variável na taxa inicial de reação. Os resultados apresentados sugerem que o aumento do tempo de reação ocasione o aumento das taxas de evaporação de água no meio reacional. Para minimizar este problema, Feiten et al. (2014) sugerem a utilização do sistema batelada alimentada.

\section{CONCLUSÃO}

Neste trabalho a obtenção de hidrolisado do óleo de Crambe assistida por irradiação ultrassônica é reportada. Os resultados obtidos relatam melhores rendimentos com adição de $40 \%$ de água (em relação à massa de óleo) e temperatura de $45^{\circ} \mathrm{C}$. O percentual de catalisador favorece a reação até percentuais de $10 \%$ (em relação à massa de substratos), sendo que acima deste percentual não é observada diferença significativa no rendimento ( $p>0,05)$, para as reações conduzidas em 4 horas. $\mathrm{O}$ efeito do tempo reacional relatou que as reações conduzidas com tempos acima de 2 horas não ocasionam o aumento no teor de AGLs.

\section{REFERÊNCIAS}

AOCS. AMERICAN OIL CHEMISTS' SOCIETY. Official methods and recommended practices. United States: Editora Champaign, 1998.

AWADALlAK, J.A., VOLL, F., RIBAS, M.C., SILVA, C., CARDOZO-FILHO, L., SILVA, E.A. Enzymatic catalysed palm oil hydrolysis under ultrasound irradiation: Diacylglycerol synthesis. Ultrason. Sonochem., v.20, p.1002-1007, 2013.

ANTCZAK, M. S.; KUBIAK, A.; ANTCZAK, T.; BIELECKI, S. Enzymatic Biodiesel Synthesiskey Factors Affecting Efficiency of The Process. Renewable. Ener., v.34, p. 1185-1194, 2009.

FEITEN, M. C.; ROSA, C. D.; TREICHEK, H.; FURIGO Jr, A.; ZENEVICZ, M. C.; OLIVEIRA, D.; OLIVEIRA, J. V. Batch and Fed-Batch Enzymatic Hydrolysis of Soybean Oil Under Ultrasound Irradiation. Biocatal. Agric. Biotech., v.01, p.01-03, 2014.

HAAS, M. J.; MCALOON, A. J.; YEE, W. C.; FOGLIA, T. A.. A Process Model to Estimate Biodiesel Production Costs. Bioresour. Technol., v.97, p.671-678, 2006.

LIU, Y.; JIN, Q.; SHAN, L.; LIU, Y.; SHEN, W.; WANG, X. The Effect of Ultrasound on Lipasecatalyzed Hydrolysis of Soy Oil in Solvent-free System. Ultrason. Sonochem., v.15, p.402-407, 2008.

MACIEL, A. M.; MING, C.; RIBEIRO, A. P. B.; SILVA, R. C.; GIOIELlI, L. A.; GONÇALVES, L. A. G. Phisicochemical Properties of Interesterified Blends of Fully Hydrogenated Crambe abyssica Oil and Soybean Oil. J.Am. Oil Chem. Soc. v.91, p. 111-123, 2014.

MENG, Y.; WANG, G.; ZHOU, Z.; LI, Y.; LIANG, X.; CHEN, J.; LI, Y.; LI, J. Two-step Synthesis of Fatty Acid Ethyl Ester From Soybean Oil Catalyzed by Yarrowia Lipolytica Lipase. Biotech for. Biofuels., v.04, p.01-09, 2011. 
OLIVEIRA, E. C.; SILVA, P. R.; RAMOS, A. P.; ARANDA, D. A. G.; FREIRE, D. M. G. Study of Soybean Oil Hydrolysis Catalyzed by Thermomyces lanuginosus Lipase and Its Application to Biodiesel Production via Hydroesterification. Enzy. Res., v.2011, p.01-08, 2011.

RANGANATHAN S. V.; NARASIMHAN S. L.; MUTHUKUMAR, K. An Overview of Enzymatic Production of Biodiesel. Bioresour. Technol., v.99, p.3975-3981, 2008.

RASPE, D. T.; CARDOZO FILHO, L.; SILVA, C. Effect of Additives and Process Variables on Enzymatic Hydrolysis of Macauba Kernel Oil (Acrocomia aculeate). Chemic. Enginee., v.2013, pp.01-08, 2013.

RIBEIRO, B. D.; COELHO, M. A.; BARRETO, D. W. Production of Concentrated Natural Betacarotene from Buriti (Mauritia vinifera) Oil by Enzymatic Hydrolysis. Food and Bioprod. Process., v.90, n.2, p.141-147, 2012.

ROONEY, D.; WEATHERLY, L. R. The Effect of Reaction Conditions Upon Lipase Catalysed Hydrolysis of High Oleate Sunflower Oil in a Stirred Liquid-liquid Reactor. Process. Biochem., v.36, p.947-953, 2001.

SILVA, M. A.; BIOAGGIONI, M. A. M.; SPEROTTO, F. C. S.; BEZERRA, P. H. S.; BRANDÃO, F. J. B. Qualidade do Óleo Bruto de Grãos de Crambe (Crambe abyssinica Hochst) Sob diferentes Métodos de Secagem. Energ. Agric., v.28, p.193-199, 2013.

THANK, L. T.; OKITSYU, K. O.; SADANAGA, Y.; TAKENAKA, N.; MAEDA, Y.; BANDOW, H. Ultrasound-assisted Production of Biodiesel Fuel From Vegetable Oils in a Small Scale Circulation Process. Bioresour. Technol., v.101, p.639-645, 2010.

YU, D., TIAN, L., WU, H., WANG, S., WANG, Y., MA, D., FANG, X. Ultrasonic Irradiation With Vibration for Biodiesel Production from Soybean Oil by Novozym 435. Process. Biochem., v.45, p. 519-525, 2010. 